\title{
High-resolution measurement technology for the detection of complex process influences in machining operations
}

\author{
Till-Hendrik Hage ${ }^{1, *}$, Marco Fritzsche ${ }^{2}$, Sebastian Henkel ${ }^{1}$, Jens Bliedtner ${ }^{1}$, \\ ${ }^{1}$ Ernst-Abbe University of Applied Sciences Jena, Carl-Zeiss-Promenade 2, 07745 Jena, Germany \\ ${ }^{2}$ Polytec GmbH, Polytec-Platz 1-7, 76377 Waldbronn, Germany
}

\begin{abstract}
Inorganic non-metallic materials are widely used in many application areas such as precision optics, semiconductor technology, high-temperature technology or medical technology. This increasingly requires the application of precise multi-axis CNC machine technology and poses great challenges for the machining processes, especially with regard to the economic production of high-quality surfaces. In the field of optics technology, high surface qualities in the nanometer range are often required. The production machines designed for this purpose must therefore guarantee a high degree of accuracy and be constant with respect to their environmental factors. An influence on the quality of the machining results that should not be underestimated is the vibrations occurring on the machine, which are caused on the one hand by the operating vibrations generated by the running spindle, and on the other hand by resonance frequencies. A side effect of these vibrations can also be damage to the workpiece or the tool. In order to detect these influences, a modal analysis and a measurement of the operating vibration mode are carried out on an ultrasonic-assisted CNC grinding machine using a three-dimensional laser Doppler vibrometer system provided by Polytec $\mathrm{GmbH}$. In addition, corresponding measurements are carried out by means of a one-dimensional laser Doppler vibrometer on a 5-axis CNC universal milling machine in order to establish comparability between an ultrasound-assisted and a conventional processing machine.
\end{abstract}

\section{Introduction \& Methods}

In general, modal analyses serve the purpose of investigating the dynamic behavior of oscillatory systems with regard to their natural frequency, natural mode and modal mass. Since vibrations occur on the workpiece and tool side during machining in addition to the set variables feed rate, cutting speed and depth of cut, they directly influence the process and thus the process result. Basically, vibrations occur in a machine tool either from the cutting process through self-excitation or through external excitation mechanisms such as unbalance, drive and gear vibrations or through neighboring machines. [2] Especially in the field of optical technology, which deals with the production of complex components made of brittle-hard materials such as fused silica or the manufacture of monolithic components, high surface qualities and accuracies in the nanometer range are required. In order to find out the influences of these resulting vibrations, investigations on an ultrasound-supported $\mathrm{CNC}$ grinding machine "Ultrasonic 20 linear" from company DMG Mori are presented below in the form of a three-dimensional modal analysis using a scanning laser Doppler vibrometer system. To enable a 3-dimensional measurement, three laser doppler vibrometers are positioned in an isosceles triangle in front of the machine. The position of the measuring devices is determined by triangulation during calibration, so that a spatial image is generated. (see Figure 1). The Doppler vibrometers measure vibrations without contact, no additional mass on the measuring object is necessary, so that a non-invasive measurement is made by means of a laser beam.

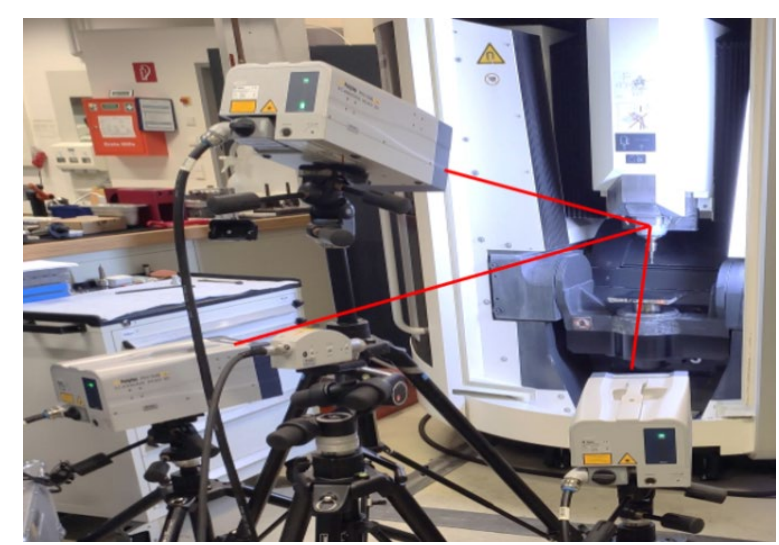

Fig. 1. Measurement setup of a Laser Doppler interferometer from Polytec GmbH

\footnotetext{
* Corresponding author: till-hendrik.hage@eah-jena.de
} 
When the laser beam comes into contact with a moving object, the back-reflecting laser beam undergoes a frequency or phase shift based on the principle of the Doppler effect. Using a heterodyne Mach-Zehnder interferometer and digital decoder technology, this frequency or phase shift is first recorded by a photodetector before being digitized and then demodulated by a digital decoder. As a result, the displacement (phase demodulation) and the velocity (frequency demodulation) of the moving object is measured over time by the Laser Doppler Vibrometer (see Figure 2).

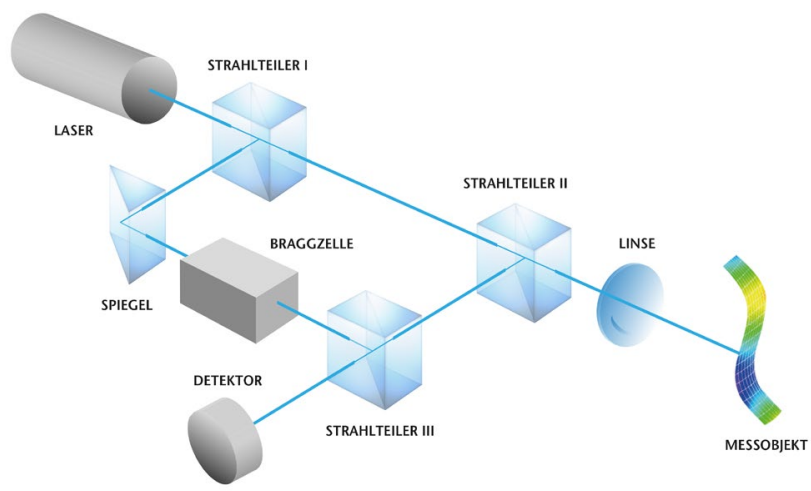

Fig. 2. Basic principle and schematic structure of a Laser Doppler interferometer from Polytec GmbH [3]

By an integrated camera and two scanning mirrors it is possible to define any number of measuring points on the object (see Figure 3). Then these measuring points are approached one after the other during the measurement. At each measuring point, the vibrations are recorded and then displayed in the evaluation in an animated vibration form with the video image superimposed. The measuring system ensures a high bandwidth and dynamics, as even high-frequency vibrations are reliably recorded and adapted to the existing amplitude of the vibration by means of different measuring ranges. The same measuring procedure is carried out on a CNC universal milling machine "DMU 50" from the company DMG Mori AG using a onedimensional scanning laser Doppler vibrometer, with the measuring direction in the $\mathrm{Y}$-axis.

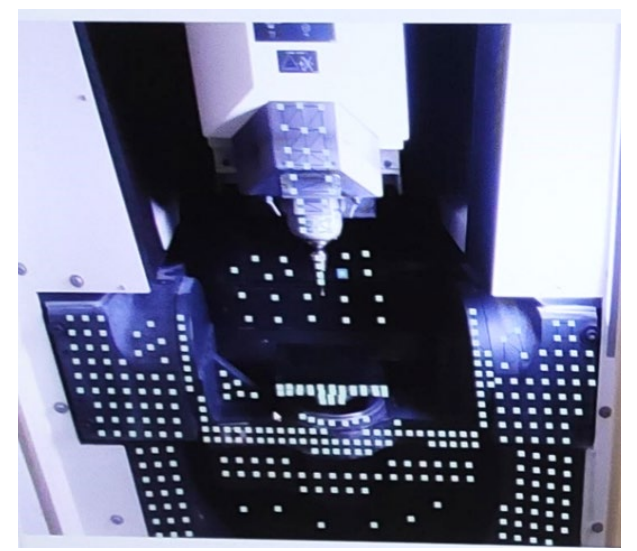

Fig. 3. Overview of measuring points, which were set individually in the object

\section{Examinations \& Results}

The results of the investigations are presented below. The ultrasonic CNC grinding machine "Ultrasonic 20 linear" has a speed spectrum of $0-40,000$ revolutions per minute. For the detection of dominant vibrations, the speed is adjusted in steps of thousands. The vibration detection of the operational deflection shape is carried out by measuring the conspicuous amplitudes and vibration frequencies on the machines. By varying the speed, a comprehensive overview is obtained. To facilitate the evaluation, this is graphically supported by visualizing the occurring vibration through the previously set measuring points in an exaggerated way (see figure 4).

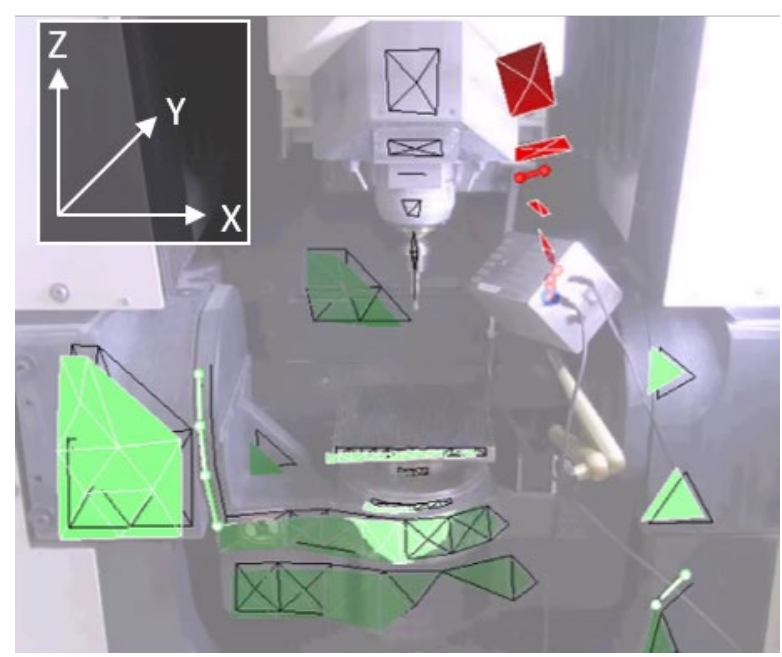

Fig. 4. Example of a measurement image for the evaluation of an occurring vibration

The strongest amplitudes in the frequency spectrum are defined as dominant vibrations. These are always to be noted at the respective natural frequencies of the speed values. For example, at a set speed of $24,000 \mathrm{rpm}$, the strongest amplitude is at $400 \mathrm{~Hz}$. When considering all measurements, a proportional influence of the set speed on the resulting amplitude can be observed, apart from the superimposition with distinct amplitude peaks (see Figure 5). However, the maximum elongation smaller than $0.1 \mu \mathrm{m}$ is to be considered small.

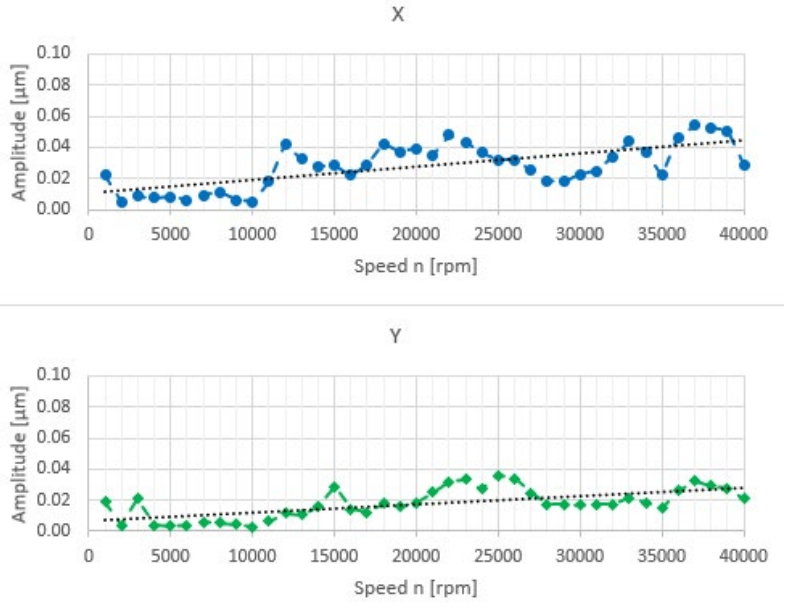




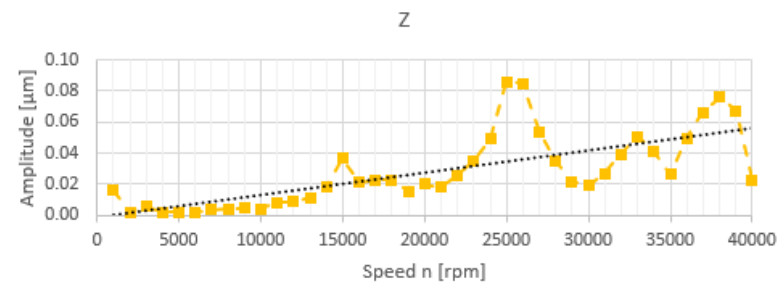

Fig. 5. Operational deflection shape: dominant vibrations in relation to speed of "Ultrasonic 20 linear"

In addition to the dominant oscillations, continuous oscillations can be noted, for example, an amplitude at 42.2 Hz, $112.5 \mathrm{~Hz}$ as well as $300 \mathrm{~Hz}$ occurs in all measurements. The latter frequency is due to vibrations of the machine table, the origin of the other two frequencies is unknown so far. Since the "Ultrasonic 20 linear" enables machining with an ultrasonic oscillation on the tool, it is also checked whether the induced vibrations have an effect on the spindle. For this purpose, the ultrasonic working frequency of the tool used is examined at varying rotational speeds in the spectrum mentioned. Continuous amplitudes can be observed over the entire speed range, which, however, do not represent significant vibration transmissions to the spindle due to their small size of less than $1 \mathrm{~nm}$.

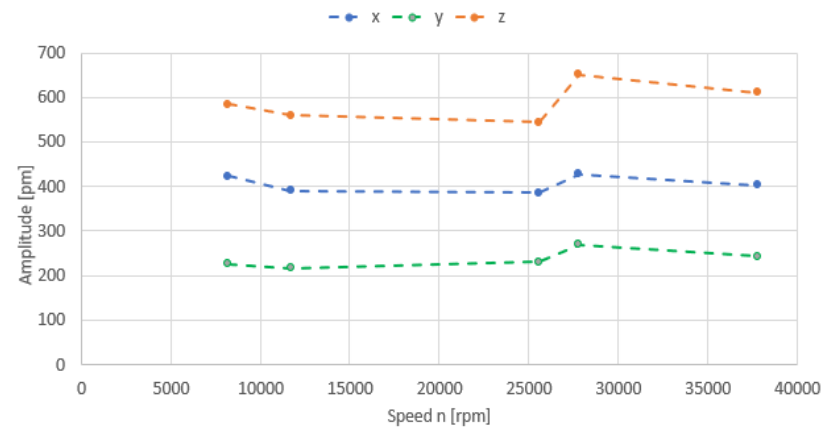

Fig. 6. Comparison of US frequency $(25.4 \mathrm{kHz})$ at different rotation speeds of "Ultrasonic 20 linear"

To perform the modal analysis, resonant frequencies are determined using a modal hammer excitation and a Dirac pulse triggered by it; a scalable automated hammer is used to avoid variable excitations. Since the previous operating vibrations occurred at the spindle and at the table, excitation is performed at both locations. The resonant frequency determined here occurs at the spindle at $136 \mathrm{~Hz}$, which corresponds to a speed of approx. $8000 \mathrm{rpm}$ and should therefore be avoided for minimal spindle vibrations. The modal analysis with excitation at the table results in a resonant frequency at $490 \mathrm{~Hz}$ and thus shows a difference to the frequency determined in the operating mode $(300 \mathrm{~Hz})$. This is presumably due to a difference in the way the measurement is carried out, since the operating mode is determined with mains voltage switched on, while the modal analysis is determined without mains voltage and therefore without compressed air.

The measurements are repeated analogously with a scanning vibrometer on the CNC universal milling machine "DMU 50". This has a speed spectrum of 0 -
$14,000 \mathrm{rpm}$, the speed is varied here in steps of 2,000 . Here too, an increase in amplitude is evident with an increase in the set speed. The maximum amplitude in space exhibits a maximum elongation of less than 1.0 $\mu \mathrm{m}$. In comparison with the "Ultrasonic 20 linear", it is clearly visible that the vibration amplitudes of the "DMU $50 "$ are approx. a factor of 10 higher. Thus, despite its higher adjustable speed, the "Ultrasonic 20 linear" achieves lower vibration amplitudes than the "DMU 50".

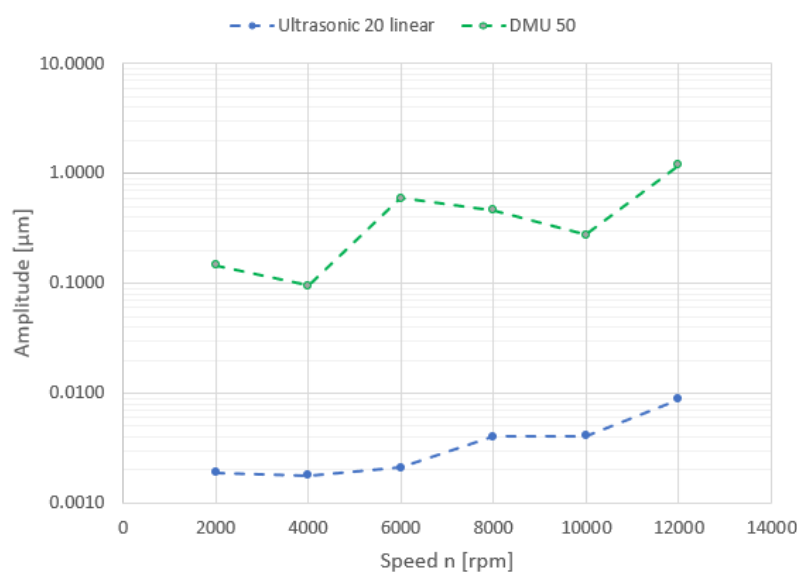

Fig. 7. Comparison of operational deflection shape of "Ultrasonic 20 linear" and "DMU 50"

\section{Conclusion and Outlook}

It was shown that higher vibration amplitudes are generated with increasing speed. These are very low for the "Ultrasonic 20 linear" with a value of less than 0.1 $\mu \mathrm{m}$. The ultrasonic vibration generated on the tool is not significantly transmitted to the spindle or the entire system. The comparison between the two processing machines shows that the "Ultrasonic 20 linear" generates 10 times lower vibration amplitudes than the "DMU 50" despite its higher adjustable speeds. In the future, the modal analysis is to be tested in process operation. For this purpose, investigations will be carried out on a new type of simultaneous measuring system consisting of a vibrometer, a force measuring platform and a thermographic camera enabling a time-synchronized measurement of three main process parameters: vibrations, forces and temperatures. In addition, the influences of the vibrations on the workpiece and the quality of the machining will be further examined.

\section{References}

1. M. Möser: Modalanalyse. Berlin: Springer Vieweg. Fachwissen Technische Akustik (2020)

2. H.K. Tönshoff, U. Wasmann: Auswirkung erzwungener Schwingungen auf die Standzeit beim Stirnfräsen. In: Forschung im Ingenieurwesen 58, pp. 283-289 (1992)

3. Polytec GmbH (2020): In Natur und Technik : Alles schwingt! [online] https://www.polytec.com/de/vibr ometrie/technologie/laser-doppler-vibrometrie 